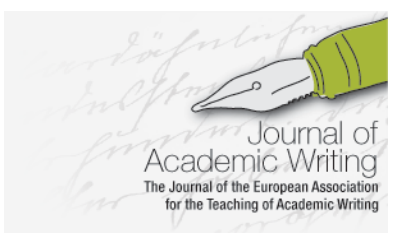

Journal of Academic Writing

Vol. 8 No 2 Winter 2018, pages 176-190 http://dx.doi.org/10.18552/joaw.456

\title{
What Can Screen Capture Reveal About Students' Use of Software Tools When Undertaking a Paraphrasing Task?
}

\author{
Carol Bailey \\ University of Wolverhampton, UK \\ Jodi Withers \\ University of Wolverhampton, UK
}

\begin{abstract}
Previous classroom observations, and examination of students' written drafts, had suggested that when summarising or paraphrasing source texts, some of our students were using software tools (for example the copy-paste function and synonym lookup) in possibly unhelpful ways. To test these impressions we used screen capture software to record 20 university students paraphrasing a short text using the word-processing package on a networked PC, and analysed how they utilised software to fulfil the task. Participants displayed variable proficiency in using word-processing tools, and very few accessed external sites. The most frequently enlisted tool was the synonym finder. Some of the better writers (assessed in terms of their paraphrase quality) availed themselves little of software aids. We discuss how teachers of academic writing could help students make more efficient and judicious use of commonly available tools, and suggest further uses of screen capture in teaching and researching academic writing.
\end{abstract}

\section{Introduction}

This observational study was prompted by our impression, gained over several years and in various contexts, that when writing on a computer - and especially when writing from sources - some of our students use software tools in ways we might not expect, or necessarily recommend. For example, when teaching in an IT lab or supporting students one-to-one, we have observed that some students appear unfamiliar with word processing software, and do not make full use of all the features at their disposal (an issue noted twenty years ago by Susser 1998). We have seen some users of English as an Additional Language (EAL), while working with source material, run large chunks of text through translation software rather than attempting to read and understand the document in English (Wrigley 2017: 7, Xu and Ding 2014: 88).

While giving formative feedback on draft assignments uploaded via Turnitin, we have noticed that, in some cases, the drafting process appears to involve pasting chunks of source material into a working document and 'tweaking' them. The 'tweaks' can involve deletion, addition, reordering sentences in a paragraph or phrases within a sentence, and (commonly) substituting words from the source text with synonyms, not all of which are aptly chosen (Howard 1993: 233, Stapleton 2010: 302, Li and Casanave 2012: 171-172). Reference to the source text may be omitted. More recently, we have witnessed a rise in the use of free online paraphrasing tools, as discussed by Rogerson and McCarthy (2017). This had come to our attention through students asking whether we endorsed their use of these tools, and via staff referrals of students for extra language support because the resulting 'word salad' is impossible for an assessor to decode. 
The aim of this study is to explore in more detail what software tools (if any) students use when paraphrasing a set text on the computer. We chose paraphrase as the focus of our investigation since this is a key skill in writing from sources which often presents a challenge to students, especially EAL users and novice writers (Cumming, Lai and Cho 2016).

The introduction of computers in education settings in the late 1970s/early 1980s inspired a wealth of research into computer-aided writing instruction. Some of this focussed on the creation and evaluation of specific writing programmes (Palmquist 2003: 396-398). More germane to our present study are investigations into the use of word-processing software, which increased in number from the early to mid-1980s, before being superseded in the 1990s by studies on collaborative writing, new media and the development of Online Writing Labs. However, the topic continues to be of interest to teachers of academic writing. The ongoing development of grammar/style and spelling checkers has prompted several scholars to revisit these tools and their pedagogic uses (Buck 2008, Figueredo and Varnhagen 2006, McGee and Ericsson 2002, Potter and Fuller 2008, Vernon 2000). Investigations which compare the processes of composing by hand or on a computer explore their respective effects on the cognitive process (Medimorec and Risko 2016), early writing outcomes (Wollscheid, Sjaastad and Tømte 2016), and the implications for time-constrained assessments (Hunsu 2015, Mogey and Fluck 2015, Whithaus, Harrison and Midyette 2008). While research in the latter field assumes a widespread familiarity with word-processing and the supersession of handwriting with keyboarding skills, it also acknowledges that not all students are equally comfortable composing on a computer. Socioeconomic inequities may play a role in this (Madden 2014).

In addition to witnessing the advent of increasingly affordable personal computers, the 1970s also saw a shift in writing research focus from product (written output/artefact) to process (Abdel Latif 2008: 30). This emphasis on what actually happens when writers compose kindled a new interest in synchronous and asynchronous observation techniques. Some methods require selfreporting by participants: questionnaires (Susser 1998), think-aloud protocol (Li 2006), stimulated recall (Bosher 1998), and process logs (Stapleton 2010). Researcher-oriented methods include documenting observations in the form of narrative field notes, or ticking predefined boxes (Hyland 2016: 118). Less subjective techniques involve the use of technology to record the writing process. While earlier studies used external video recorders to film participants writing (Matsuhashi and Cooper 1978), the mid-1980s saw the introduction of programmes which could capture activity on the computer itself (Abdel Latif 2008: 31-32): namely keystroke logging (Spelman Miller 2005) and screen capture - also known as screen recording - software (Elola and Mikulski 2013).

Since each method has particular strengths and drawbacks - for example, computer recording programmes cannot capture writers' use of offline tools - many scholars use two or more techniques to triangulate their findings. Leijten and Van Waes (2013) discuss complementary methods, giving as an illustration the combination of keystroke-logging and eye-tracking software to investigate the process of reading while writing. Park and Kinginger (2010) used a combination of corpus query logs, screen capture and oral/written reflections to explore their participant's linguistic decision-making process. In their investigation of L2 writing in test- and non-test conditions, Khuder and Harwood (2015) used a mixed-methods approach involving keystroke-logging (to measure time allocation), screen capture software (to assist stimulated recall), field notes (enabled through Skype screen-sharing by the participants), and analysis of the texts produced.

Keystroke logging, which documents not only keyboard and mouse activity but also pages visited, has received more attention in the writing process literature to date, but affords a less user-friendly visualisation of the writing process than screen capture does (Khuder and Harwood 2015: 245, Park and Kinginger 2010: 34, Xu and Ding 2014: 80-81). Since our research question does not necessitate the microsecond analysis functionality afforded by keystroke-logging programs, we elected to use screen capture software in our study, as it is very easy to incorporate recordings obtained by this method into student learning resources and teacher development materials. We chose Snagit (2018) as a mid-priced and wellsupported package whose functionality best met our needs. 


\section{Method}

\section{Participants}

Our twenty participants were recruited from courses on which we teach, ${ }^{1}$ and from an extracurricular Academic Integrity online course. The students were drawn from a broad spread of subject areas at $\mathrm{FHEQ}^{2}$ levels 3-8, i.e. Foundation courses to PhD study, and ranged in age from 19 to 51. Of the ten males and ten females, eight were native English speakers, and twelve were users of English as an Additional Language. In brief exit interviews we asked our participants two questions concerning their normal practice when drafting an assignment:

1. Do you usually draft on paper or on a computer? Why?

2. Are there any special software tools you normally use?

Beyond asking students about their normal drafting process, we did not control for computing skills, as our purpose was simply to observe participants' use of software tools rather than to compare the proficiency of different groups.

\section{Writing task}

Participants were asked to paraphrase one of two short texts on a PC. The source texts were taken from Wikipedia, were 90 words long and had Flesch-Kincaid Grade Levels of 12 and 12.2. ${ }^{3}$ To avoid the topic familiarity effect (Khuder and Harwood 2015: 242), we chose topics (cell phone use and CCTV monitoring) which we thought would be familiar and interesting to all students, irrespective of their academic discipline. Our main considerations in determining text length and readability were (i) as our participants were volunteers, we should not take up too much of their time; (ii) we wished to include students on the International Foundation Year (pre-degree level), who enter their course with an English level of IELTS 5.5. English language entry requirements for UK undergraduate study average IELTS $6.0-6.5$, and we wanted to make sure the texts we chose were accessible to participants just below this level as well as to PhD students and native English speakers.

Since we had only two single-user licenses for our screen capture software (Snagit 2018), participants undertook the computer paraphrase on our office PCs - i.e. not in their normal composing environment. We started and stopped the screen capture at the beginning and end of each writing episode. One participant accidentally turned off the recording software early in the task, leaving us with 19 videos in total.

\section{Data analysis}

We independently viewed two videos to generate and agree our coding categories; the remaining 17 were initially coded by one researcher and checked by the other. The codes and their definitions are outlined in Table 1.

\footnotetext{
${ }^{1}$ In a post-1992 university (former polytechnic) in England.

${ }^{2}$ Framework for Higher Education Qualifications in England, Wales and Northern Ireland.

${ }^{3}$ Computed in 2015 by www.readability.com - this site has since been discontinued. FleschKincaid Grade Levels can also be accessed under the 'readability' settings in Microsoft Word's spelling and grammar checker.
} 
tools in their writing session. The protocol for the task instructed participants to use Microsoft Word as they usually would, and all participants used Microsoft Word's default settings.

Table 2. Use of software tools in the video paraphrasing task

\begin{tabular}{lcc}
\hline Software tool & $\begin{array}{c}\text { Total number of } \\
\text { times used }\end{array}$ & $\begin{array}{c}\text { Proportion of total tool use } \\
\text { ( \% ) }\end{array}$ \\
\hline External sources & 36 & $15 \%$ \\
Grammar check: interacts with tool & 30 & $12 \%$ \\
Grammar check: self-corrects & 7 & $3 \%$ \\
Context menu & 20 & $8 \%$ \\
Spellcheck: interacts with tool & 54 & $22 \%$ \\
Spellcheck: self-corrects & 30 & $12 \%$ \\
Synonym finder & 56 & $23 \%$ \\
Thesaurus & 11 & $5 \%$ \\
& & $100 \%$ \\
\hline Total number of times tools used: & 244 & \\
\hline
\end{tabular}

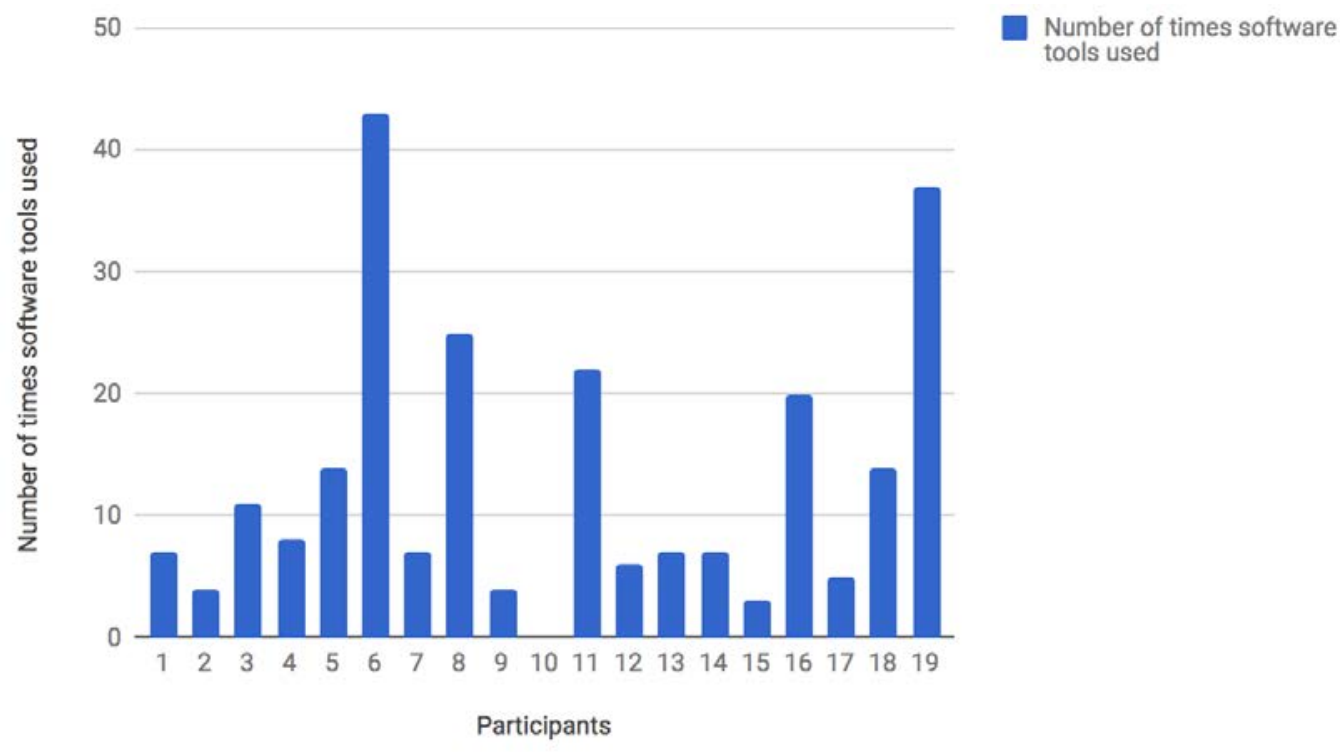

Fig. 1 Number of times each participant used software tools

However, these data somewhat obscure the individual differences we observed in how the tools were used. We give examples below.

\section{Green and red squiggly lines}

A widely-recognised tool built into Microsoft Word's default proofing settings is the red and green 'squiggly' lines which flag in real time that a spelling or grammar/style error has been made. Our participants responded to the squiggly lines in different ways: by self-correcting (editing the text manually, without further tool use) or by exploring the software options to investigate and resolve the error. Tools were typically accessed by right-clicking on the flagged section to call up a context menu. We found no instances of 'false positives' in the sections flagged by Word: i.e. they were in fact errors.

\section{Responding to the red and green squiggly line: Self-correcting}

In $36 \%$ of cases where the red squiggly line appeared, participants self-corrected. In contrast, participants self-corrected in 19\% of cases where the green squiggly line flagged an error. This could suggest that participants were more confident in correcting spelling, or that a proportion of the spelling mistakes were due to mistyping rather than lexical knowledge. 


\section{Use of the grammar check tool}

Participants used the grammar checker to resolve mistakes in the majority of cases where a green squiggly line indicated a problem (30 times out of 37). Several cases were due to mistyping, for example, unnecessary spaces.

However, this does not mean that their interactions with the grammar checker were unproblematic. One example of this is Esther, ${ }^{5}$ who struggled to implement the tool's feedback. Esther, an EAL user, had written the sentence fragment Even though all cultures don't use it in the same way, which was flagged with a green squiggly line. Responding to the green line, she right-clicked on the sentence to open up a context menu, which advised: Fragment (consider revising). She right-clicked to open and close a series of menus in an apparently random manner, and eventually selected the option 'About this sentence'. This opened a new window containing advice about fragments, which she closed after a short pause. After opening and closing two more context menus in quick succession, Esther deleted the word even, before revising though to Though. The green line persisted. Finally, she selected Ignore Once, which immediately removed the green line. She moved on to a new sentence and did not return to the fragment. Esther therefore made no attempt to alter the structure of the sentence, perhaps indicating that the advice given by the tool was not understood.

\section{Use of the spellcheck tool}

The spellcheck was the second most-used software tool. Typically, participants right-clicked on the incorrect spelling to open up a menu displaying correct alternatives. Clicking on a suggestion automatically replaced the incorrect spelling. Spellcheck was used to correct mistakes in $64 \%$ of cases where spelling errors were flagged by a red squiggly line. However, not all participants used the tool: seven out of nineteen opted to self-correct only.

We observed the spellcheck being used with varying degrees of efficiency. One participant, Robert, used it on 15 occasions (only self-correcting once), immediately after keying in a misspelled word. He appeared to rely on the spellcheck to keep the writing process flowing, perhaps because using it was more efficient than retyping the word. It is worth noting that this participant is dyslexic and often miskeyed words.

In other instances, the spellcheck was used inefficiently, causing disruption to the writing flow. For example, if a word was misspelled to the point where the software algorithm could not identify it, the spellcheck was of limited use, so the participant had to resolve the error in another way. In one extended sequence, the participant (Ada) keyed in ackownlegemenof. After making small unsuccessful revisions, she right-clicked on the letter string twice to call up the context menu, which did not display a suggestion until she inserted a space to make two recognisable words (ackownledgment and of). The menu then displayed the correct spelling, which Ada selected. Interestingly, no participants used software tools for a final spelling and grammar check at the end of their writing session.

\section{Finding Synonyms}

As might be expected for a paraphrase writing task, the MS synonym finder represents the greatest proportion of digital tool use, comprising $23 \%$ of all interactions with software tools. We therefore analysed in more detail how it was used. In $38 \%$ of cases, the participant made no selection after viewing the list of suggestions. We evaluated $34 \%$ of the synonyms selected as appropriate choices, for example infer for extrapolate or district for jurisdiction. However, $29 \%$ of the synonyms chosen were coded as unsuitable (did not collocate/usage problem/wrong connotation/wrong meaning/wrong register), e.g. unrestricted as a synonym for public (wrong connotation) or convert for become (wrong meaning). Only a quarter of the words selected were located at the top of the list of suggestions: this could indicate that participants were fairly discriminating when choosing synonyms. However, we also observed instances where synonym selection appeared to be mechanical and undiscriminating. An example is the segment, In the past few years, society has become less tolerant of cell phone use in public areas. In one extreme case, the participant (Omar) paraphrased this as, In the previous years, community has convert fewer in accepting the use of telephone in unrestricted spaces. While

${ }^{5}$ Pseudonyms are used throughout, to protect our participants' identity. 
the synonyms previous and community were typed by Omar himself, convert, fewer, accepting, telephone, unrestricted and spaces were selected from the MS synonym finder. Omar applied this process of synonym substitution throughout his paraphrase.

We also noted that, in $54 \%$ of cases, participants searched for synonyms by clicking on words in the source text, while in $46 \%$ of cases, they searched for the synonym within their paraphrase. However, in 50/56 instances the word they selected came from the source text; i.e. participants who used the synonym finder appeared to be resorting to this tool instead of drawing on their own lexical repertoire. It is worth mentioning that only one participant (Joanne) used Microsoft Word's built-in thesaurus, with the rest of the students opting to right-click on the word to access the synonym finder. She accessed it early in the writing process [ 1 minutes 55 seconds] and kept it open for the duration of her writing [total writing time 12 minutes 22 seconds], using it 11 times.

\section{External Sources}

We analysed instances where external online sources (for example, online dictionaries) were accessed. Only three participants used external sources, with one participant being responsible for half of all interactions.

An example of how one student (Katrin) used external sources illustrates how adept digi-literate students can be. Katrin used an online German-English dictionary (linguee.com) and two websites to establish a suitable synonym for the word recognition (a process that took her approximately 1 minute and 51 seconds). Firstly, after viewing the synonym finder's suggestions for recognition, she opened up an internet tab and keyed anerkennung into the address bar. This defaulted to a Google search, where she clicked on the top link, synonmye.woxikan.de/synonyme/anerkennung.php. After scrolling down the page for several seconds, Katrin went to linguee.com (already open) and keyed in the word popularitaet. The web-page refreshed to display populatität and its English translation popularity. Returning to her paraphrase, Katrin used popularity in her next sentence.

\section{Use of other features within Microsoft Word}

Eight participants were observed using formatting features within MS Word to manipulate both the source text and their paraphrase. These included using bold (1), ${ }^{6}$ changing the font (1), highlighting (3), underlining (1), cutting and pasting text (3), changing background colour (1), and using brackets (1). One participant opened up a new Word document and, keeping both the source text and the new document visible, typed their paraphrase into the new file. Towards the end of their writing session, they cut and pasted their paraphrase into the source text document.

\section{Discussion}

\section{Preference for paper or computer as a writing medium}

At a brief exit interview, we asked students whether they normally drafted university assignments on paper or on the computer. Eight participants said they usually drafted on paper; nine preferred drafting on a computer; and three said they used both. Seven out of nine students who preferred drafting on a computer mentioned speed as a key affordance: one student said he could type faster than he could write; another said that note-taking on a computer avoided duplication of effort (transferring paper notes). Five students found it quicker to edit their text on a computer, and three said it was easier to plan and (re)organise their document. Neatness was a factor for three students, who found drafting on paper was 'scruffy' with 'lots of crossingsout'. Other affordances mentioned were the spellcheck (2), ability to look up words (2), and ability to highlight text for future editing. Finally, having source material, writing tools and the writer's notes/drafts all in one place was seen by two students as an advantage: 'on the computer, everything's there' and 'everything is electronic these days'.

\footnotetext{
${ }^{6}$ Numerals in brackets indicate the number of participants.
} 
In contrast, eight of our twenty participants said they preferred drafting on paper. Some found this medium easier for making notes (3), paraphrasing (2), or proofreading (2). Regarding proofreading, one participant remarked, 'If I'm writing on the computer I don't see any mistake or errors and submit it straight away'. One student from a rural part of Nigeria, where the electricity supply was irregular and IT/internet access a rare luxury, told us he had been writing on paper 'since my childhood'. Keyboarding skills were a factor mentioned by two: one mature student from the UK explained that he had to look at the keyboard while typing, and found this distracted him from the composing process (cf. Barkaoui 2016). For this participant, handwriting was an automatic process: 'I can think ahead at the same time [...] I don't need to look at the paper when I'm writing'. Two other students mentioned a cognitive benefit of handwriting: one felt 'more connected to ideas'; the other said 'it gives me time to think'. In this case the relative slowness of writing by hand was seen as an affordance, as with Mogey and Fluck's participant (2015: 800): 'Pen and paper means you can think about the next step as you are writing. Typing, I only think as far as the sentence I am writing and I am concerned with spelling mistakes'.

Our participants' preferences reflect those expressed by university students in recent research by Fortunati and Vincent (2014: Italy), Taipale (2014: Finland) and Farinosi, Lim and Roll (2016: Germany, Italy and the UK). In the latter study, preference varied according to context (with a computer being the preferred composing medium for academic coursework assignments); however all students appreciated the computer's affordances of automatic error correction, access to tools such as synonym finder and dictionaries, and editing functions such as copy/cut/paste to facilitate the modification of written content. In Finland (Taipale 2014), students were critical of their word-processor's autocorrect functions (possibly due to Finnish linguistic factors) but appreciated its affordances of speed, ease of editing and textual productivity. At the same time, participants in all three studies enjoyed the versatility, portability, immediacy and cognitive space offered by paper.

\section{Use of software tools}

At the exit interview, we asked participants if there were any special software tools they normally used for composing on a computer. Responses ranged from 'None', through MS Word spellcheck and synonym finder, to external tools such as online dictionaries and thesauri. Six students mentioned using Google to search for definitions and synonyms. More specialist tools included Grammarly, Refworks, text magnification software, and the online 'paraphrasing' applications spinbot.com and paraphrasing-tool.com.

Our screen recordings revealed participants' variable proficiency in the use of software tools. While some struggled to operate the spellcheck or became distracted by green squiggly lines (cf. Buck 2008: 409-410), others used tools discriminatingly and in interesting ways. The limited functionality and intrusive effect of MS spelling and grammar/style checkers have been critiqued by several authors (Vernon 2000), as have their ideological and pedagogical implications (McGee and Ericsson 2002), including their potential for foregrounding surface corrections over more meaningful revisions (Buck 2008). Against this are studies such as that by Figueredo and Varnhagen (2006), who found that use of these tools during the revision process increased the number and accuracy of surface corrections, while not impacting negatively on content revisions. ${ }^{7}$ While recognising the limitations of built-in word-processing tools, we concur with scholars who advocate their place in the writing classroom (Potter and Fuller 2008), in one-toone writing tutorials (Buck 2008) and when giving written feedback on student drafts (Stapleton and Radia 2010).

Potter and Fuller's engaging account of a four-month action research project demonstrates how critical use of a grammar checker within a seventh-grade language arts curriculum in the US empowered Potter's students and made them both more confident and sceptical in their utilisation of the tool, sparking 'truly investigative discussion' (2008: 39) of grammar and style. The role of such tools as a 'springboard for conversation' is also propounded by Vernon (2000: 344), who likewise advocates teaching students how to customise the software options and

\footnotetext{
${ }^{7}$ It should be noted that, in this study, participants were proofreading texts composed by a third party rather than composing their own.
} 
gives several suggestions for classroom use. ${ }^{8}$ In a case study of two individual writing tutorials, Buck (2008: 412) observes that neither tutor nor student participants took full advantage of the range of tools available within MS Word and online. She paints a scenario in which a writing tutor might discuss with the student how best to manage the grammar checker, use Track Changes as a revision record for later review, highlight text to flag up areas for further work, employ copy-paste to explore alternative textual organisation, and play with layout to demonstrate issues such as sentence length.

\section{Vocabulary development}

Similarly, students can be encouraged to make better use of the lexical tools at their disposal. When we presented our preliminary findings at EATAW 2017, some delegates suggested that writing teachers should discourage students from accessing shortcuts such as the MS synonym finder, and focus on vocabulary development instead. While we agree that a structured, scaffolded approach to vocabulary acquisition is key in an intensive English language or academic writing course, most of our participants were not enrolled in such courses and in several cases were juggling academic coursework deadlines with employment or childcare commitments, further restricting their access to English language/writing support. It is understandable that students in this situation will avail themselves of tools which are instantly available, round-the-clock. As writing teachers - and in the interests of learner autonomy (Conroy 2010: 861-863, Lin, Pandian and Jaganathan 2017) - we feel it incumbent on us to promote more judicious use of such aids. Surely our goal should be to discourage the indiscriminate and mechanical use of synonym substitution exhibited by Omar, while fostering the dictionary search techniques so ably deployed by Katrin.

While semantically inappropriate choices made up the majority of synonym fails in our study (39/60 in the computer condition), we do not have space here to do justice to the wealth of literature concerning dictionary use (for a recent research timeline, see Nesi 2014). Regarding the MS Word synonym tool, it is very easy (and fun!) to create mini-lessons exploring the limitations of this tool. Word class is one issue to explore: failure to identify the class of a suggested synonym may lead to confusion for EAL users (cf. Nesi and Haill's 2002 findings on dictionary consultations). For example, a synonym search in MS Word 2010 for 'used' in the phrase 'back translation has been used' (verb: present perfect passive) returns the suggestions 'rummage-sale, second-hand, cast-off, hand-me-down, recycled,' (adjectives). While native English speakers may automatically discard such inappropriate suggestions because they 'feel' wrong, even they may benefit from a review of word class in order to make fuller use of MS Word/online thesaurus functions where parts of speech (POS) identifiers are used.

Synonym context is another issue to explore with students. Though inappropriate collocation occurred in only five of our sixty synonym fails, it may be useful for students to explore why 'firmly banned' does not work as a synonym for 'strictly prohibited'. Students to whom we have introduced Phil Edmond's JustTheWord collocation finder (http://www.just-the-word.com/) ${ }^{9}$ tell us they find it very helpful and user-friendly, especially for checking preposition and synonym collocates. Conroy (2010) explores how online concordancing tools can help students develop their understanding of lexico-grammatical patterns (in our sample, usage problems accounted for another 5/60 synonym fails). Conroy extends his discussion of concordancing to include intelligent Google searches as a form of corpus query, finding that several participants preferred Google to concordancers due to its larger corpus and greater flexibility.

Another lexical software tool to consider is machine translation. MS Word now includes this function in a range of languages, and translation engines such as Google Translate are only a mouse click away. Writing teachers might encourage EAL students to make more judicious use of these tools rather than banning them completely. While back translation has been deployed as a device for 'cheating' text-matching software such as Turnitin (Jones and Sheridan 2014), writing teachers could use this method to illustrate the pitfalls of whole-text translation

\footnotetext{
${ }^{8}$ For example: exploring the performance of a specific grammar check function; correcting flagged errors without recourse to the tool's suggestion; deliberately creating 'bad' sentences to see whether the grammar/style checker can recognise and correct the flaws.

${ }^{9}$ Based on the British National Corpus.
} 
compared with single word/phrase lookup, or exploit the app's flawed output as a prompt for discussing more correct/appropriate alternatives (Nino 2009).

Notwithstanding the utility of software tools in vocabulary development, even strong advocates advise caution. For EAL users with lower levels of English proficiency, the plethora of alternatives presented by dictionaries and thesauri may be overwhelming (Motteram 2013: 35). Several studies have shown that web search and dictionary consultation while composing can interrupt the writer's 'flow', particularly for those with a lower English proficiency (Xu and Ding 2014). Teachers might consider encouraging students to refrain from accessing lexical tools during the composing process, postponing their use to the revision stage. As Stapleton and Radia point out (2010: 178), 'in many cases, it would be only the most ambitious student that would rigorously use all the tools mentioned.' In the interests of efficiency as well as learner autonomy, we should encourage our students to be critical and selective in the software tools they employ.

\section{Relationship between paraphrase quality and use of software tools}

As we observed our participants' variable proficiency in software tool use and evaluated their written output, we asked: is it possible to be a good writer without the assistance of software tools? We computed a Pearson product-moment correlational coefficient to assess the relationship between amount of tool use and quality of paraphrase. ${ }^{10} \mathrm{~A}$ non-significant weak negative correlation of $r(19)=.-164(p=n . s)$ was found. Although this result is not statistically significant, it is worth highlighting the individual variation observable in the participants' scores, against the number of times they accessed tools. One participant who scored $8 / 10$ used tools 43 times, while another who also scored 8/10 used them five times. Our highest scoring participant $(9 / 10)$ used tools only four times. This suggests that it is possible for students to write well without software aids.

\section{Conclusion}

Our screen recordings revealed many fascinating insights into how participants approached the paraphrasing task which, due to considerations of space and focus, we are not able to report here. Screen capture has received less coverage than keystroke logging as a method in writing process research, possibly because keystroke logging allows for greater precision in measuring temporal aspects such as pause length. However, the added functionality of screen capture as a teaching and learning tool commends its use to teachers of academic writing. Recordings of student writing can be used to prompt reflection in a tutorial context or (with the student's permission) readily be repurposed as learning resources. We have also found them a powerful visual aid, both at external conferences and internal staff development events, for raising colleagues' awareness of how learners write.

One key finding from our study was that some student writers do not make full or efficient use of the software tools available to them, and would benefit from instruction in these. In particular, writing teachers could help them manage and critically evaluate the language aids embedded in their word-processing package, as there is no added cost implication in using these. At the same time, our findings show that it is possible to be a good writer (and produce a competent paraphrase) without using any software tools beyond the typing interface.

Our study has some limitations. Firstly, our participants were self-selected. Nine volunteered as a gesture of goodwill, while eleven took part because they wanted advice on their academic writing/paraphrase skills. For these reasons - and possibly also because they were being observed - they exhibited none of the behaviours we have noted in other contexts (copying chunks, block translation, use of online 'paraphrasing' tools). They may not have typified the range of behaviours and motives displayed by university students completing a written

10 The combined scores for conceptual equivalence and coherence on the Paraphrase Evaluation Tool. 
assignment, often under time pressure and sometimes with little interest in or understanding of the task topic.

Secondly, the set texts we asked our participants to paraphrase may not be representative of the source types university students are expected to draw on, which characteristically have lower readability scores and are frequently on unfamiliar topics. Research shows that the readability of a document influences the level of copying when students paraphrase (Sun 2012), so our results may have been very different had we chosen more difficult, specialist or longer passages.

Finally, our controlled writing task (paraphrasing a set text) gives only a tiny glimpse of what students actually do when searching for and utilising sources in a written assignment. As Stapleton (2010) suggests, different methods are required to investigate the processes involved in a typical 3000-word student assignment, composed over a period of weeks in various locations, and drawing on multiple sources.

Our study suggests several avenues for further research using screen capture software to investigate student writing. Using the same task with a different sampling strategy and wider participant base might yield different insights into software use and paraphrasing behaviour. A similar task with more difficult, specialist or longer source texts might give different results. A less controlled task (for example a time-constrained essay) would show how students search for and incorporate source texts in their writing. Screen capture software could also be harnessed as a video diary in tracking the completion of an actual student assignment - this would lend itself to a case study approach. The creative use of screen capture software has the potential to illuminate many aspects of student writing, not least how the digital world and writing processes interact.

\section{Acknowledgments}

We would like to thank our colleague Anastasia Novoselova, and delegates at the Solstice/CLT Conference (Edge Hill University, June 2016), for trialling our Paraphrase Evaluation Tool, and delegates at EATAW 2017 for their insightful comments on our preliminary findings. We are particularly grateful to the anonymous reviewers of our initial article submission for their detailed and constructive comments. 


\section{References}

Abdel Latif, M. M. (2008) 'A State-of-the-Art Review of the Real-Time Computer-Aided Study of the Writing Process'. International Journal of English Studies 8 (1), 29-50

Bailey, C., and Withers, J. (unpublished) 'Paraphrasing on a Computer or by Hand: What are the Differences?'

Barkaoui, K. (2016) 'What and When Second-Language Learners Revise when Responding to Timed Writing Tasks on the Computer: The Roles of Task Type, Second Language Proficiency, and Keyboarding Skills'. The Modern Language Journal 100 (1), 320-340

Bosher, S. (1998) 'The Composing Processes of Three Southeast Asian Writers at the PostSecondary Level: An Exploratory Study'. Journal of Second Language Writing 7 (2), 205-241

Buck, A. M. (2008) 'The Invisible Interface: MS Word in the Writing Center'. Computers and Composition 25 (4), 396-415

Conroy, M. A. (2010) 'Internet Tools for Language Learning: University Students Taking Control of their Writing'. Australasian Journal of Educational Technology 26 (6), 861-882

Cumming, A., Lai, C., and Cho, H. (2016) 'Students' Writing from Sources for Academic Purposes: A Synthesis of Recent Research'. Journal of English for Academic Purposes 23, 47-58

Edmonds, P. (n.d.) 'Just the Word'. [online] available from <http://www.just-the-word.com/> [14 November 2017]

Elola, I., and Mikulski, A. (2013) 'Revisions in Real Time: Spanish Heritage Language Learners' Writing Processes in English and Spanish'. Foreign Language Annals 46 (4), 646-660

Farinosi, M., Lim, C., and Roll, J. (2016) 'Book or Screen, Pen or Keyboard? A Cross-Cultural Sociological Analysis of Writing and Reading Habits Basing on Germany, Italy and the UK'. Telematics and Informatics 33, 410-421

Figueredo, L., and Varnhagen, C. K. (2006) 'Spelling and Grammar Checkers: Are They Intrusive?' British Journal of Educational Technology 37 (5), 721-732

Fortunati, L., and Vincent, J. (2014) 'Sociological Insights on the Comparison of Writing/Reading on Paper with Writing/Reading Digitally'. Telematics and Informatics 31 (1), 39-51

Howard, R. (1993) 'A Plagiarism Pentimento'. Journal of Teaching Writing 11 (3), 233-46

Hunsu, N. J. (2015) 'Issues in Transitioning from the Traditional Blue-Book to Computer-Based Writing Assessment'. Computers and Composition 35, 41-51

Hyland, K. (2016) 'Methods and Methodologies in Second Language Writing Research'. System 59, 116-125

Jones, M., and Sheridan, L. (2014) 'Back Translation: An Emerging Sophisticated Cyber Strategy to Subvert Advances in 'Digital Age' Plagiarism Detection and Prevention'. Assessment and Evaluation in Higher Education 40 (5), 712-724

Khuder, B., and Harwood, N. (2015) 'L2 Writing in Test and Non-Test Situations: Process and Product'. Journal of Writing Research 6 (3), 233-278 
Leijten, M., and Van Waes, L. (2013) 'Keystroke Logging in Writing Research: Using Inputlog to Analyze and Visualize Writing Processes'. Written Communication 30 (3), 358-392

$\mathrm{Li}$, J. (2006) 'The Mediation of Technology in ESL Writing and its Implications for Writing Assessment'. Assessing Writing 11, 5-21

Li, Y., and Casanave, C. P. (2012) 'Two First-Year Students' Strategies for Writing from Sources: Patchwriting or Plagiarism?' Journal of Second Language Writing 21 (2), 165180

Lin, D. T. A., Pandian, A., and Jaganathan, P. (2017) 'Facilitating Learner Autonomy: Reading and Effective Dictionary Use for Lexical Development'. The Reading Matrix [online] 17 (2), 40-51. available from <http://www.readingmatrix.com/files/17-lj107025.pdf> [30 October 2017]

Madden, S. (2014) 'Obsolescence in/of Digital Writing Studies'. Computers and Composition $33,29-39$

Matsuhashi, A., and Cooper, C. (1978) A Video Time-Monitored Observational Study: The Transcribing Behavior and Composing Processes of a Competent High School Writer. [online] available from <https://files.eric.ed.gov/fulltext/ED155701.pdf> [15 November 2018]

Medimorec, S., and Risko, E. F. (2016) 'Effects of Disfluency in Writing'. British Journal of Psychology 107 (4), 625-650

McGee, T., and Ericsson, P. (2002) 'The Politics of the Program: MS Word as the Invisible Grammarian'. Computers and Composition 19 (4), 453-470

Mogey, N., and Fluck, A. (2015) 'Factors Influencing Student Preference When Comparing Handwriting and Typing for Essay Style Examinations'. British Journal of Educational Technology 46 (4), 793-802

Motteram, G. (ed.) (2013) Innovations in Learning Technologies for English Language Teaching. [online] available from <https://www.teachingenglish.org.uk/> [16 November 2017]

Nesi, H. (2014) 'Dictionary Use by English Language Learners'. Language Teaching 47 (1), 3855

Nesi, H., and Haill, R. (2002) 'A Study of Dictionary Use by International Students at a British University'. International Journal of Lexicography 15 (4), 277-305

Nino, A. (2009) 'Machine Translation in Foreign Language Learning: Language Learners' and Tutors' Perceptions of its Advantages and Disadvantages'. ReCALL 21 (2), 241-258

Palmquist, M. (2003) 'A Brief History of Computer Support for Writing Centers and WritingAcross the-Curriculum Programs'. Computers and Composition 20 (4), 395-413

Park, K., and Kinginger, C. (2010) 'Writing/Thinking in Real Time: Digital Video and Corpus Query Analysis Language'. Language, Learning and Technology 14 (3), 31-50

Potter, R., and Fuller, D. (2008) 'My New Teaching Partner? Using the Grammar Checker in Writing Instruction'. English Journal 98 (1), 36-41

Rogerson, A. M., and McCarthy, G. (2017) 'Using Internet-Based Paraphrasing Tools: Original Work, Patchwriting or Facilitated Plagiarism?' International Journal for Educational Integrity 13 (2) 1-15 
Snagit (2018) The Leader in Screen Capture Software. [online] available from $<$ https://www.techsmith.com/screen-capture.html> [15 November 2018]

Spelman Miller, K. (2005) 'Second Language Writing Research and Pedagogy: A Role for Computer Logging?' Computers and Composition 22, 297-317

Stapleton, P. (2010) 'Writing in an Electronic Age: A Case Study of L2 Composing Processes'. Journal of English for Academic Purposes 9 (4), 295-307

Stapleton, P., and Radia, P. (2010) 'Tech-Era L2 Writing: Towards a New Kind of Process'. ELT Journal 64 (2), 175-183

Sun, Y. C. (2012) 'Does Text Readability Matter? A Study of Paraphrasing and Plagiarism in English as a Foreign Language Writing Context'. Asia-Pacific Education Researcher 21 (2), 296-306

Susser, B. (1998) 'The Mysterious Disappearance of Word Processing'. Computers and Composition 15 (3) 347-371

Taipale, S. (2014) 'The Affordances of Reading/Writing on Paper and Digitally in Finland'. Telematics and Informatics 31, 532-542

Vernon, A. (2000) 'Computerized Grammar Checkers 2000: Capabilities, Limitations, and Pedagogical Possibilities'. Computers and Composition 17, 329-49

Whithaus, C., Harrison, S. B., and Midyette, J. (2008) 'Keyboarding Compared with Handwriting on High-Stakes Writing Assessment: Students' Choice of Composing Medium, Raters' Perception, and Text Quality'. Assessing Writing 13 (1), 4-25.

Wollscheid, S., Sjaastad, J., and Tømte, C. (2016) 'The Impact of Digital Devices vs. Pen(cil) and Paper on Primary School Students' Writing Skills: A Research Review'. Computers and Education 90, 19-35

Wrigley, S. (2017) 'Avoiding 'De-plagiarism': Exploring the Affordances of Handwriting in the Essay Writing Process'. Active Learning in Higher Education [online] available from <https://doi.org/10.1177/1469787417735611> [30 October 2017]

Xu, C., and Ding, Y. (2014) 'An Exploratory Study of Pauses in Computer Assisted EFL Writing'. Language Learning and Technology 18 (3), 80-96 


\section{Appendix 1: Paraphrase Evaluation Tool}

\begin{tabular}{|c|c|c|}
\hline Mark & Conceptual equivalence & Coherence \\
\hline 5 & $\begin{array}{l}\text { All information in the source text is fully } \\
\text { and effectively represented. There are no } \\
\text { omissions or changes in meaning. }\end{array}$ & $\begin{array}{l}\text { Language use is sophisticated and } \\
\text { versatile, with no errors. Transformations } \\
\text { are skilful and do not draw attention to } \\
\text { themselves. }\end{array}$ \\
\hline 4 & $\begin{array}{l}\text { Most information in the source text is } \\
\text { competently represented. There may be } \\
\text { small changes in meaning and/or some } \\
\text { minor omissions. }\end{array}$ & $\begin{array}{l}\text { There may be some minor errors, e.g. in } \\
\text { spelling or punctuation, but these do not } \\
\text { reduce the communicative effect. A few } \\
\text { transformations may appear clumsy or } \\
\text { laboured. }\end{array}$ \\
\hline 3 & $\begin{array}{l}\text { Most information in the source text is } \\
\text { adequately represented. The meaning } \\
\text { may be distorted in some places and/or } \\
\text { some points are omitted. }\end{array}$ & $\begin{array}{l}\text { Some errors in syntax and word choice } \\
\text { but these do not prevent the message } \\
\text { from coming through. }\end{array}$ \\
\hline 2 & $\begin{array}{l}\text { Information in the source text is } \\
\text { incompletely represented. Meaning may } \\
\text { occasionally be lost due to } \\
\text { misunderstanding or language errors. } \\
\text { Some key points are omitted. }\end{array}$ & $\begin{array}{l}\text { Several errors in syntax and word choice, } \\
\text { rendering some parts of the paraphrase } \\
\text { difficult to understand. }\end{array}$ \\
\hline 1 & $\begin{array}{l}\text { Information from the source text is } \\
\text { inadequately conveyed, due to } \\
\text { significant changes in meaning and/or } \\
\text { severity of language errors. Large } \\
\text { sections of text may be omitted. }\end{array}$ & $\begin{array}{l}\text { Many errors in syntax and word choice } \\
\text { prevent the meaning from coming } \\
\text { through. }\end{array}$ \\
\hline
\end{tabular}

